\title{
AMYLOIDOSIS
}

\section{FIVE CASES OF PRIMARY GENERALIZED AMYLOIDOSIS AND SOME OTHER UNUSUAL CASES \\ BY}

\author{
W. St. C. SYMMERS
}

From the Department of Morbid Anatomy; Charing Cross Hospital and Medical School, London

A conception of amyloidosis as merely a complication of chronic syphilitic, tuberculous or septic disease of bones and joints or of chronic intrathoracic sepsis is no longer adequate. This older view has had to be modified as the association of amyloidosis with other diseases came to be recognized: the predisposing diseases are now known to include rheumatic and rheumatoid affections, myelomatosis, Hodgkin's disease, ulcerative colitis, pyelonephritis and a variety of other conditions. In addition, the occurrence of generalized amyloidosis in the absence of any recognized predisposing cause, that is, " primary amyloidosis," has lately attracted increasing attention. The first indisputable cases of this sort were reported by Soyka (1876) and Wild (1886), but the condition did not begin to be widely known until after Lubarsch (1929) defined certain pathological differences between this atypical form of amyloidosis and ordinary secondary amyloidosis. These differences have proved to be neither absolute nor diagnostic, although they characterize a majority of the cases: in fact, it is now known that there is no one feature which occurs exclusively in any form of amyloidosis. How far primary amyloidosis may be considered an entity remains undecided, but the present tendency is to consider all forms of amyloidosis to be closely related.

Various aspects of the history, pathology, aetiology, and clinical pictures of amyloidosis, with special reference to primary amyloidosis, have been reviewed elsewhere in this issue. In the present paper five cases of primary amyloidosis are recorded, and some cases illustrating other unusual manifestations and associations of amyloidosis are described briefly.

\section{Cases of Primary Generalized Amyloidosis}

Case 1 : Primary Amyloidosis Presenting with Progressive Heart Failure.-A previously healthy builders' foreman, aged 61 , began to be short of breath on ordinary exertion, such as hurrying about his business, and this symptom became slowly severer. About a year later his feet and ankles began to swell, and during the next nine months he gradually developed widespread oedema and effusions in the serosal cavities. His doctor found mercurial diuretics and full doses of digitalis to be without effect on these symptoms, even after repeated tapping of the pleural and peritoneal effusions. Two years after the onset of dyspnoea the patient had become so incapacitated that he at last agreed to go to hospital.

On admission he was orthopnoeic, cyanotic, and dropsical. The heart was enlarged and gallop rhythm was present. The pulse ( 86 per minute) was regular: the blood pressure was $130 / 70 \mathrm{~mm}$. of mercury. There was gross ascites, a large pleural effusion on the right and a small one on the left. The liver was slightly enlarged.

Investigations. -Urine: protein +++ : occasional hyaline casts. Blood: erythrocytes $4,600,000$ per c.mm. : haemoglobin 12.0 g. per $100 \mathrm{ml}$. Serum albumin $3.0 \mathrm{~g}$. per $100 \mathrm{ml}$., globulin $3.2 \mathrm{~g}$. Blood urea $55 \mathrm{mg}$. per $100 \mathrm{ml}$. Kahn test negative. Electrocardiography showed the usual changes associated with digitalis therapy, and also low voltage in all leads.

Progress.-A litre of clear straw-coloured fluid was aspirated from the right pleural cavity and $2 \frac{1}{2}$ litres from the abdomen. Mersalyl was injected daily, and $0.25 \mathrm{mg}$. of digoxin given by mouth every six hours : there was no diuresis, his pulse rate dropped to 56 per minute within 48 hours and the beats became coupled. Treatment with digoxin was stopped, and within 24 hours auricular fibrillation developed. From this time his condition deteriorated rapidly. Fourteen days after admission he died suddenly.

Necropsy.-There was generalized dropsy, with large pleural and peritoneal transudates. The right lung was completely collapsed; the left lung was partly collapsed, hyperaemic, and oedematous. A long, coiled thrombus was impacted at the bifurcation of the main pulmonary artery. Partly adherent thrombus filled the deep veins of both calves; the thrombus on the left extended up into the external iliac vein, while that on the right had broken off in the poplitea. The heart $(610$ g.) was uniformly enlarged, with thickening of the myocardium of all parts and dilatation of all chambers. The myocardium was firm, and mottled with irregular, pale, opaque patches. The valves and endocardium were normal. 


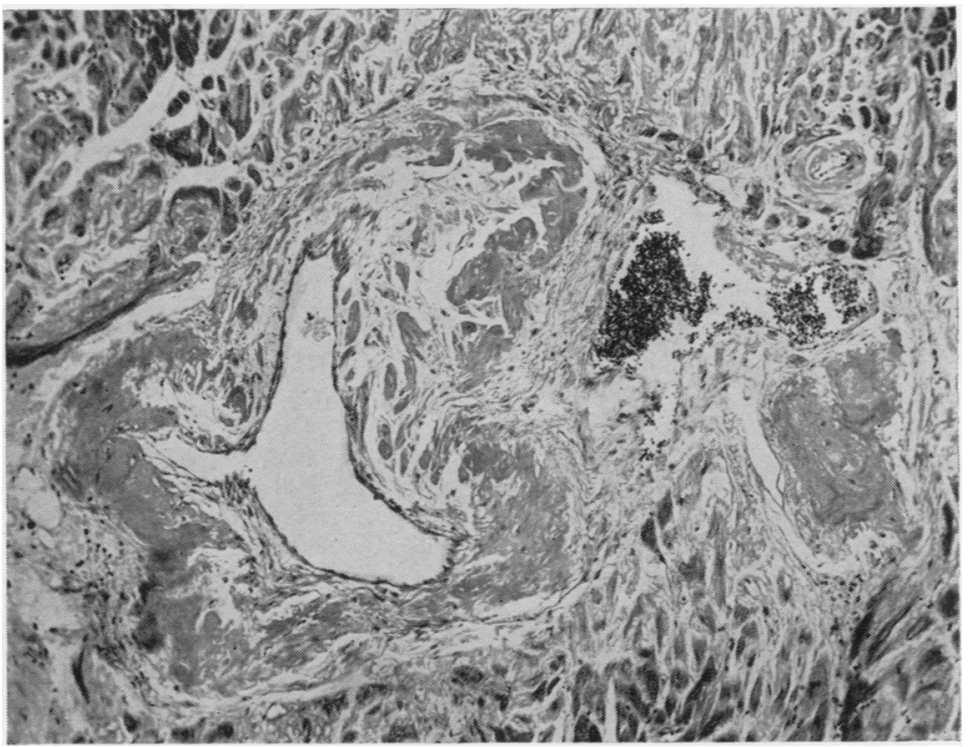

Fig 1-Case 1. Perivascular and juxtavascular amyloidosis, amyloid replacement of media of arteriole, and (top) amyloidosis of myocardium. Haematoxylin and eosin. $\times 80$.

FIG. 2.-Case 1. Large focus of myocardial cytolysis. Haematoxylin and eosin. $\times 150$.

FIG. 3.-Case 1. The most heavily involved of the very small number of glomeruli which were found to contain amyloid. Haematoxylin and eosin. $y, 350$.

Fio. 1

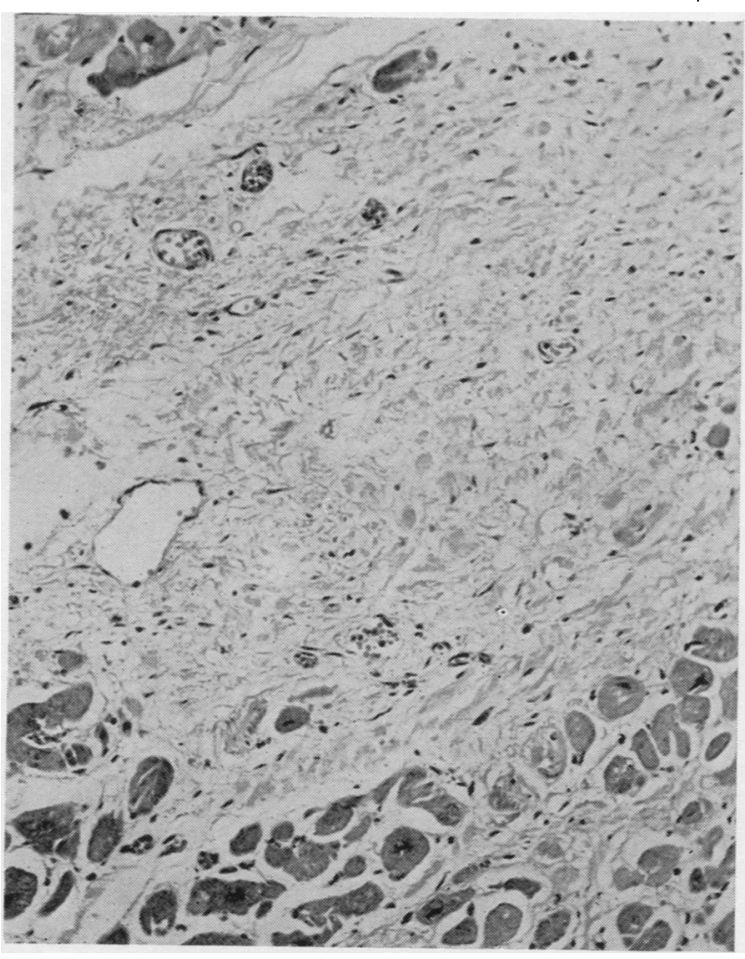

Fig. 2

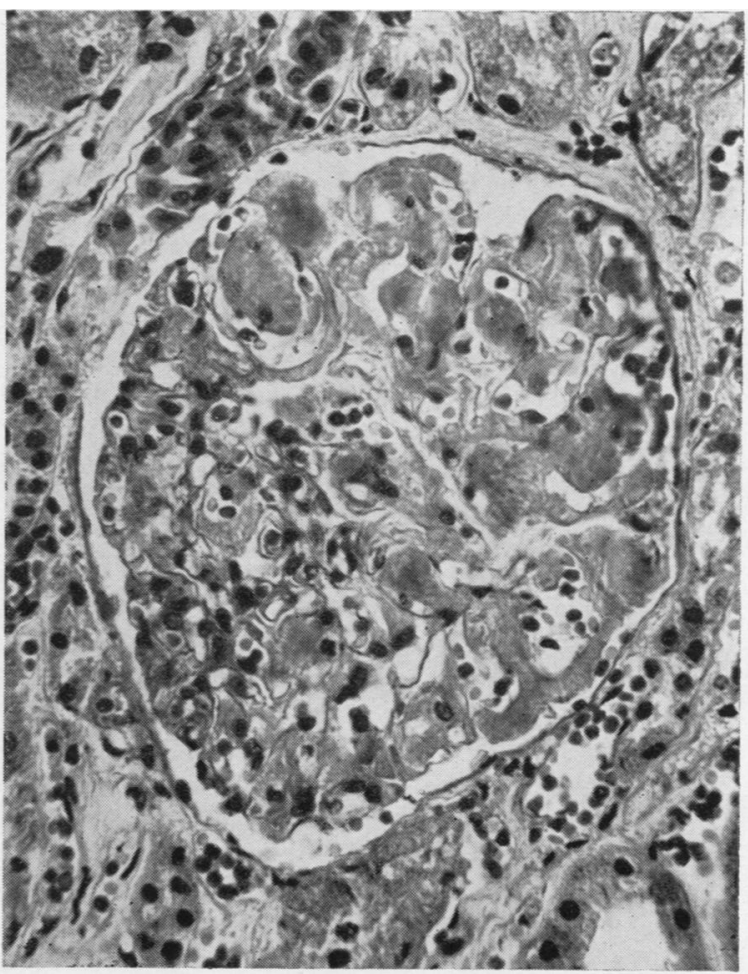

Fig. 3 
Early fibrinous pericarditis was present. The liver (1,950 g.), spleen (115 g.), and kidneys (200 and 190 g.) showed typical chronic venous congestion only. There were no noteworthy changes in other viscera or tissues, including the accessible bones.

Histology.-There was extensive amyloidosis, predominantly affecting the myocardium, but widespread also in the walls of blood vessels, particularly small arteries and arterioles, throughout many organs (Fig. 1). Extravascular deposits of amyloid were also found in the musculature of the tongue, stomach, and small intestine. and in scanty small clumps beneath the endocardium. The amyloid showed little or no affinity for Congo red, but usually gave a metachromatic reaction with methyl violet. There was no granulomatous reaction around any of the amyloid deposits. No plasma-cell proliferation was found.

The arteries most heavily affected were those in the heart and liver. There was widespread focal myocardial cytolysis (Fig. 2) and patchy interstitial myocardial fibrosis. Renal amyloidosis was limited to small deposits in occasional glomeruli (Fig. 3) and heavier involvement of interlobular and larget arteries ; apart from amyloidosis there was focal cortical atrophy, with basal glomerular hyalinization and interstitial fibrosis in these areas. In the spleen amyloid was found only in some trabecular vessels: some follicles showed interstitial hyalinization: the hyaline tissue resembled amyloid when stained with eosin, but was coloured intensely red by Van Gieson's fluid. Slight to moderate amyloidosis of blood vessels was found in the tongue, stomach, intestines, thyroid, adrenals, reproductive organs and skeletal muscle; none was found in the lungs or in adipose tissue. or in the thrombosed veins in the legs.

The main clinical feature of this case was the progressive development of cardiac failure, characteristically showing the lack of response to treatment which is a feature of amyloid heart disease. Gunnar, Dillon, Wallyn, and Elisberg (1955) found that cardiac catheterization studies confirmed the clinical observation of a similarity between the cardiovascular disturbances of myocardial amyloidosis and constrictive pericarditis; they attributed the resemblance to the lowered elasticity of the amyloid myocardium. The later stages of heart failure in the case described here were associated with massive accumulations of fluid in the serosal cavities, more suggestive of a nephrotic state than of cardiac dropsy, particularly as there was persistently heavy proteinuria : necropsy showed that there was in fact only slight renal damage. The nephrotic syndrome, which may go on to uraemic renal failure, has been observed in primary amyloidosis (Muehrcke, Pirani, Pollack, and Kark, 1955). Fatal pulmonary embolism is the only unusual feature of the present case.
Primary amyloidosis itself does not appear to predispose to thrombosis.

Case 2: Primary Amyloidosis Presenting with Heart Failure and Peripheral Neuropathy.--A dairy storeman, aged 47 , was admitted to hospital with three months history of rapidly progressive breathlessness, associated with pins-and-needles and numbness in the hands and feet. There was no history of serious illhealth previously.

On admission he was orthopnoeic and somewhat cyanotic. and his legs were oedematous. There were rales at the lung bases. Auricular fibrillation was present (heart rate about 90 per minute), and the heart was considerably enlarged. Blood pressure about $11060 \mathrm{~mm}$. of mercury. The peripheral arteries were $\omega$ not thickened; the retinal vessels were normal. There was slight loss of pain and touch sensation in the $\vec{N}$ hands and feet, with some wasting of the thenar and $\mathrm{O}$ hypothenar muscles and weakness of the grip; the $\longrightarrow$ ankle reflexes were absent and there was weakness of $\square$ plantar flexion; neurological examination was otherwise normal. The liver was slightly enlarged and $\frac{\mathscr{C}}{\mathscr{S}}$ tender. No other clinical abnormalities were found.

Investigations.-Urine: protein, a trace. Blood: erythrocytes $4,400,000$ per c.mm.; haemoglobin $10.8 \mathrm{~g}$. per $100 \mathrm{ml}$; leucocytes 8.200 per c.mm. (normal differential count). Erythrocyte sedimentation rate 110 $\mathrm{mm}$. in one hour (Westergren). Serum albumin $3.5 \mathrm{~g}$. per $100 \mathrm{ml}$., globulin $7.7 \mathrm{~g}$. Blood urea $40 \mathrm{mg}$. per $100 \mathrm{ml}$. Kahn test negative. Cerebrospinal fluid: $\stackrel{\circ}{\mathbb{D}}$ total protein $70 \mathrm{mg}$. per $100 \mathrm{ml}$.: excess globulin present (Pandy); Wassermann reaction negative; no $\overrightarrow{\overrightarrow{0}}$ increase in cells. Electrocardiography showed auri- 3 cular fibrillation; changes indicative of previous myocardial infarction were present. although there was no history of symptoms suggesting myocardial ischaemia at any time. Radiographs of the skull, ? chest, spine, and pelvis were normal.

Progress.-His condition did not respond to full doses of digitalis and vitamin-B complex. He died in $\mathrm{O}$ his sleep 10 days after admission.

Necropsy.-The tissues were waterlogged, and there $\frac{0}{3}$ were small effusions in the body cavities. The heart $D$ (640 g.) was enlarged, mainly by thickening of the ven- 으․ tricular walls and dilatation; much of the posterior $\bar{N}$ wall of the left ventricle was replaced by the fibrous $\Omega$ scar of an old infarct; there was a large pale recent $N$ infarct in the apical part of the left ventricle. The rest $\mathrm{N}$ of the myocardium was firm, and was irregularly $\underset{\sigma}{\sigma}$
mottled by pale areas. The endocardium, particularly of the atria and the ventricular aspect of the tricuspide and mitral valve cusps, was studded here and there $\mathbb{D}$ with firm, translucent nodules, about $0.2 \mathrm{~cm}$. in dia-? meter. The pericardium was normal apart from $\square$ adhesions over the old infarct. Organizing thrombus $\bar{P}$ occluded the anterior interventricular coronary artery to within about $1 \mathrm{~cm}$. of its origin; fresh thrombus $\overrightarrow{\mathbb{D}}$ extended proximally from this level and occluded the left coronary artery at its bifurcation. The distal half of the posterior interventricular artery was represented $\varnothing$ 
by a fibrous cord. The coronary arteries were otherwise normal. The aorta was slightly atheromatous. The lungs showed chronic venous congestion, with considerable basal oedema. The liver $(2,150$ g.), stomach and intestines, spleen (200 g.), kidneys (each $240 \mathrm{~g}$.), and adrenals showed typical chronic venous congestion. The brain, spinal cord, and main peripheral nerves were macroscopically normal. The cranium, spine, thoracic cage, pelvis, scapulae, clavicles, one humerus, and one femur were searched for evidence of myeloma : none was found.

Histology.-Apart from chronic venous congestion of the viscera and the myocardial infarcts, the significant histological findings were those of amyloidosis. The kidneys and bones in particular showed no other abnormalities; there was no evidence of myeloma or of plasma-cell proliferation. Amyloid was present in most of the tissues sectioned, and, apart from the subendocardial nodules, was virtually confined to the walls of the blood vessels. It stained metachromatically with methyl violet and showed strong affinity for Congo red. The vascular involvement took two forms. In one form, the commoner, the entire media of arteries and arterioles, and, less often, of veins, in various organs and tissues was replaced by amyloid, with more or less encroachment upon the lumen; there was no cellular reaction of any sort around these lesions. In the other form irregular clumps of amyloid were present in the connective tissue immediately surrounding the media of the vessels : macrophages and foreign-body giant cells were closely applied to many of these clumps, some of which were stippled with calcium.

Affected vessels were oftenest seen in the heart and in the adventitia of the aorta. There was less frequent involvement of vessels in the submucosa of the stomach and intestines, in bone marrow and around the adrenals, and rare involvement in hepatic portal tracts, pancreas, lungs, kidneys, spleen, lymph nodes, and thyroid. No amyloid was found in the reproductive organs, skeletal muscle, tongue, or skin, or in the meninges, brain, cranial nerves, or spinal cord. The spinal nerves were involved sparsely, but sections at different levels between their roots and peripheral distribution clearly indicated the likelihood of a cumulative injury through the effects of a series of amyloid vascular lesions scattered along their length.

The occluded stretches of the coronary arteries were particularly heavily affected by amyloidosis, but the amyloid was mainly in the adventitia, with only scattered deposits in the media and intima. The recent thrombosis had occurred on typical atheromatous plaques.

Heart failure in this case could be explained wholly on the basis of myocardial ischaemia caused by coronary atheroma and thrombosis, although it is likely that the widespread vascular amyloidosis played some part. It is noteworthy that atheroma was confined to the sites of actual occlusion, and that amyloidosis was particularly severe in these parts. The absence of anginal pain or of any clinical evidence of an acute infarctive episode is remarkable.

There is no reason to doubt that amyloidosis of the vasculature of the spinal nerves accounted for the neurological manifestations. Amyloid neuropathy has been reviewed by Ritama and af Björkesten (1954); Mathews (1954) cited additional cases.

The uncommonly high serum globulin level is interesting in relation to the possible pathogenetic significance of hyperglobulinaemia in amyloidosis : its cause is obscure.

Case 3: Primary Amyloidosis Presenting with Goitre and Congestive Heart Failure.--A man, aged 35 , was admitted to hospital complaining of enlargement of the neck and breathlessness. His health had been good until a year earlier, when he noticed that his collars were becoming tight. Three months later he went on an annual walking holiday and found his capacity for this exercise greatly lessened by shortness of breath. His neck continued to enlarge, breathlessness became slowly severer, and his feet began to swell in the evenings. There were no other symptoms.

On admission, examination showed a large, smooth, firm, symmetrical goitre; there was no evidence of hyperthyroidism or hypothyroidsm. His legs were oedematous and there were rales at the base of each lung. The veins of the neck were engorged, the heart was slightly enlarged and there was an apical systolic murmur. The pulse was regular (90 per minute). Blood pressure $135 / 65 \mathrm{~mm}$. of mercury. The edge of the liver was palpable just below the costal margin. The spleen reached halfway from the costal margin to the umbilicus. His tongue was uncommonly large, although otherwise normal in appearance; its mobility was decreased.

In view of the macroglossia, and heart failure without obvious cause, the diagnosis of amyloidosis was made. The patient collapsed and died suddenly on the day after admission. The only investigations which had been completed were : Urine : protein + . Blood : erythrocytes $5,500,000$ per c.mm.; haemoglobin $15.1 \mathrm{~g}$. per $100 \mathrm{ml}$; leucocytes 6,800 per c.mm. Serum albumin $3.3 \mathrm{~g}$. per $100 \mathrm{ml}$, globulin $3.8 \mathrm{~g}$. Erythrocyte sedimentation rate $58 \mathrm{~mm}$. in one hour (Westergren). Wassermann and Kahn tests negative.

Necropsy.-The pericardial sac was distended with fresh blood clot (about $400 \mathrm{ml}$.). The heart $(500 \mathrm{~g}$.) was enlarged; there was an irregular rent, about $2 \mathrm{~cm}$. long, in the wall of the left ventricle adjacent to the septum. The myocardium was extensively replaced by amyloid: the rupture had occurred between a large amyloid plaque in the anterior wall of the ventricle and an area of apparently healthy muscle. Minute amyloid nodules were scattered in the endocardium of all chambers: the valves were normal and the peri. cardium was free from obvious amyloid. The thyroid (250 g.) was symmetrically enlarged, smooth, and waxy- 


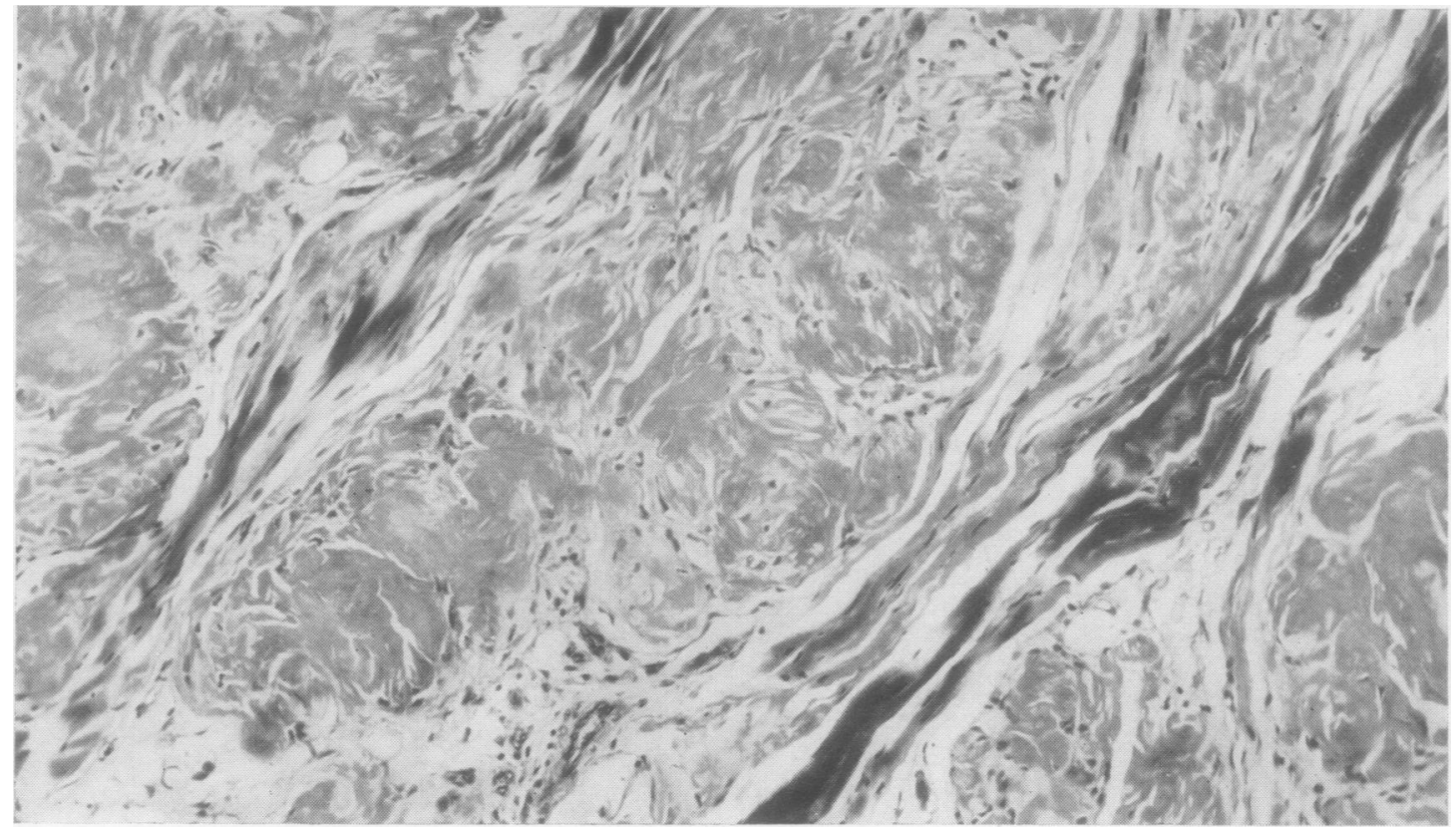

FIG. 4.-Case 3. Large amyloid deposits separating and compressing muscle fibres of tongue. Methyl violet. $\times 155$.

looking. The tongue was also heavily infiltrated with amyloid. The lungs, liver $(1,800 \mathrm{~g}$.), stomach, intestines, kidneys (combined weight, $370 \mathrm{~g}$.), and adrenals showed chronic venous congestion ; a fibroma, $0.6 \mathrm{~cm}$. in diameter, was present in a pyramid in one kidney. The spleen $(700 \mathrm{~g}$.) was practically completely replaced by amyloid. Lymph nodes in various situations were moderately enlarged.

Histology. - Widespread amyloidosis of blood vessels was found in all organs. Confluent interstitial amyloid was abundant in the myocardium, tongue (Fig. 4), thyroid, spleen, lymph nodes, and haemopoietic marrow. Pericellular amyloid was seen in adipose tissue from various sites and in the musculature of the gastrointestinal and urinary tracts, seminal vesicles, and prostate, as well as in the less heavily affected parts of the myocardium (Fig. 5) and tongue. In the myocardium there were appearances suggesting amyloid transformation of muscle cells as well as the pericellular and interstitial deposition. The liver and kidneys were free from amyloid, apart from involvement of the walls of some arteries and arterioles, and extensive interstitial deposits in the renal fibroma. The $\stackrel{\Phi}{\circ}$ lungs contained nodular masses of amyloid alongside $\overrightarrow{\vec{P}}$ heavily affected vessels; amyloid was also present in $\frac{0}{3}$ some alveolar septa, and there was considerable involvement of the bronchi, where amyloid in places merged into the cartilage and elsewhere formed nodules which jutted into 응

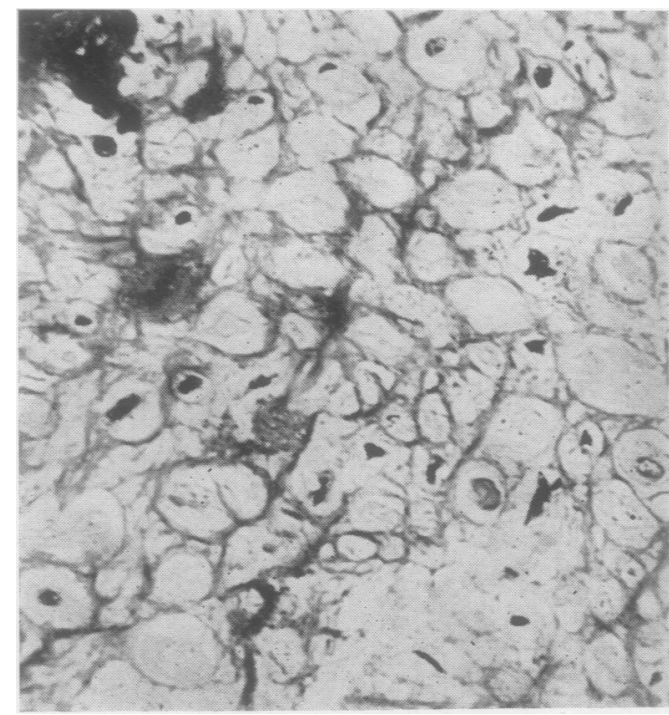

Fig. 5.-Case 3. Pericellular amyloidosis of myocardium. Congo red and haematoxylin. $\times 375$. the airway.

The amyloid had little $\stackrel{3}{3}$ or no affinity for Congo 8 red, but for the most part $३$ stained metachromatically $\mathrm{O}$ with methyl violet. A giant-cell reaction was pre- $\frac{D}{O}$ sent around the amyloid in the spleen and some lymph $N$ nodes, but not elsewhere.

Rupture of the myocardium has not been $\omega$ recorded previously in any case of amyloidosis. Spontaneous rupture of $\Phi$ the spleen has been re- + ported on three occasions $\frac{T}{\circ}$ (King and Oppenheimer, $\stackrel{\overrightarrow{\mathbb{D}}}{\mathbb{Q}}$ 1948 ; Wiley, Teeter, and $\stackrel{?}{\mathbb{P}}$ Schnabel, 1951 ; Dra- $\cong$ piewski, Sternlieb, and Jones, 1955), and patho- 


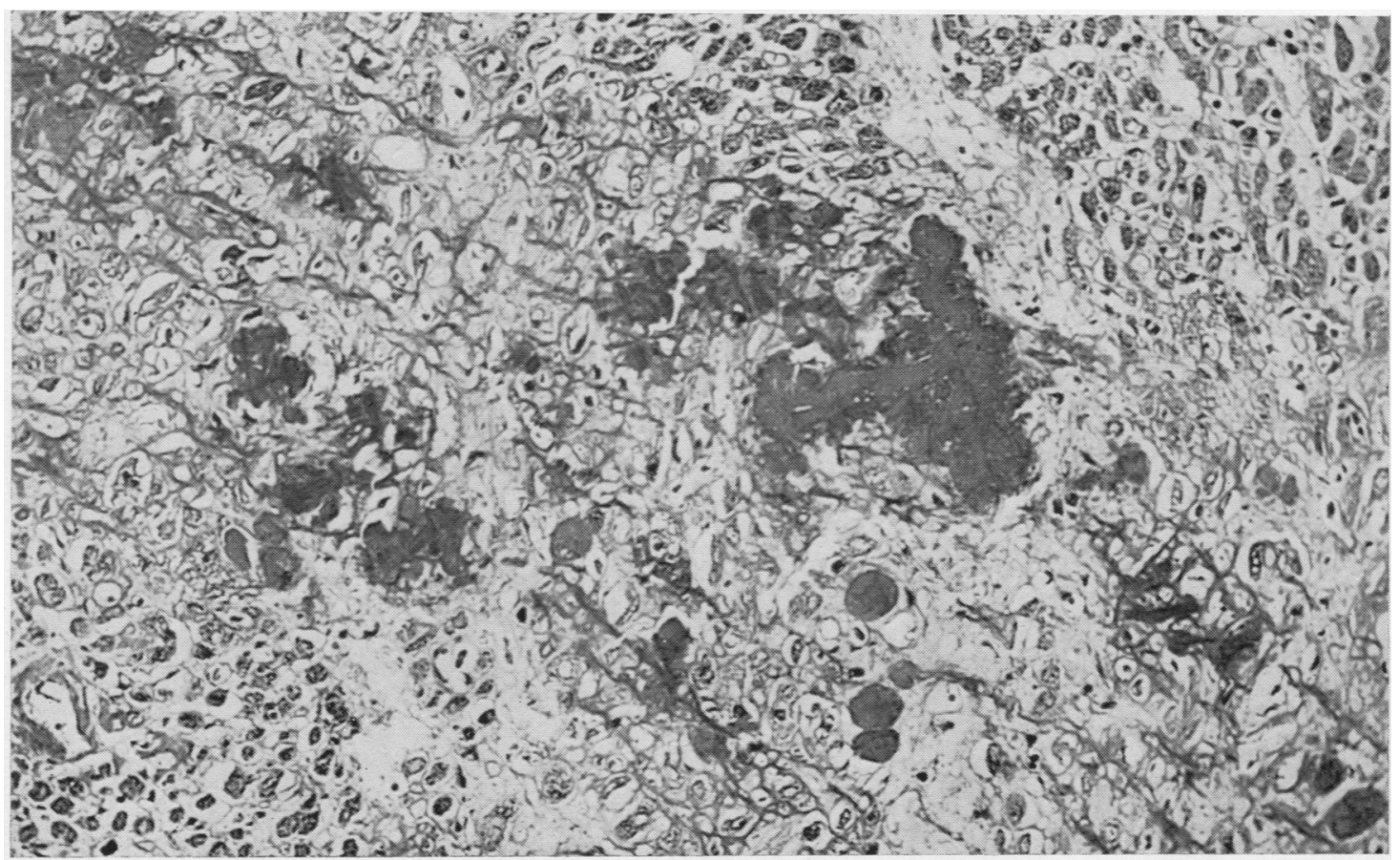

FIG. 6.-Case 4. Pericellular amyloidosis and irregular masses of amyloid in myocardium. Haematoxylin and eosin. $\times 125$.

logical fracture associated with osseous amyloidosis has been described (Koletsky and Stecher, 1939). Amyloid goitre is quite well known (Walker, 1942), usually occurring in association with chronic bronchopulmonary sepsis; the thyroid is commonly affected in primary amyloidosis, but the degree of involvement is rarely sufficient to be apparent macroscopically, and frank goitrous enlargement must be very rare.

Case 4 : Primary Amyloidosis with Obscure Symptoms and Haemorrhagic Manifestations.-A scaffolder, aged 44, complained of headaches and depression : no abnormality was found. The Wassermann and Kahn tests were negative. Six months later he was scen again, complaining of hoarseness of three months' duration and of pain in the right side of the chest, aggravated by exertion and of three weeks' duration. He had some upper abdominal pain and complained of breathlessness. Chest radiography, an opaque meal, and electrocardiography showed no abnormality. After a further six months he was admitted to hospital complaining of persistence of the pain in the abdomen and chest, hoarseness, and loss of appetite. He had had melaena for two months and had coughed up a little blood-stained sputum.

On admission he was pale and had clearly lost much weight. The edge of the liver was about $5 \mathrm{~cm}$. below the costal margin. Haemoglobin $32 \%$. Leucocytes 20,000 per c.mm. (polymorphonuclear leucocytosis). Opaque meal normal. Chest radiograph: enlargement of heart ; opacity of base of right lung. He was given 2 pints $(1,100 \mathrm{ml}$.) of blood soon after admission. Three days after admission his leucocyte count was 10,000 per c.mm., platelet count 145,000 per c.mm., and blceding time normal. His condition deteriorated quickly, crepitations developed at the base of the lungs, and he died five days after admission.

Necropsy.-There were a few petechiae on the side of the neck and on the palate. The heart $(470 \mathrm{~g}$.) showed hypertrophy of both ventricles; the myocardium was pale. The endocardium of the atria and of the atrioventricular valves was rough ; in appearance and feel it resembled the pericardium in fibrinous pericarditis. Thrombus was present in several small pulmonary arteries and there were a few small infarcts in the lungs. The lower lobe of the right lung was consolidated. Multiple submucosal haemorrhages were found in the small intestine. The liver and spleen were enlarged and congested. Nothing else of note was found.

Histology.-There was extensive and severe amyloidosis of the walls of arteries, arterioles, and veins in the myocardium and small intestine, and less involvement in the lungs, liver, and elsewhere. Pericellular amyloidosis was present in the heart (Fig. 6) and parallel striae of amyloid traversed practically the entire thickness of the atrial wall. There were extensive subendocardial and subepicardial deposits. In the intestine parts of the muscularis mucosae were wholly replaced by amyloid; large fresh haemorrhages had occurred around heavily involved submucosal vessels. 
Amyloid was present in some interalveolar septa in the lungs, but pulmonary involvement was predominantly seen in arteries, and affected both bronchial and pulmonary vessels; lobar pneumonia was present in the right lower lobe, and there were haemorrhagic infarcts elsewhere, the cause of which was not apparent in the sections. The liver, kidneys, and spleen were congested; amyloid was scanty, and confined to arteriole walls.

This case illustrates the vague symptoms which may for long be the only manifestations of primary amyloidosis. The eventual occurrence of haemorrhagic manifestations is not uncommon in primary amyloidosis, and was observed also in the following case.

Case 5: Primary Amyloidosis with Predominant Haemorrhagic and Pulmonary Manifestations.-A man, aged 47, was admitted to hospital with a history of coryza and productive cough 18 days earlier, followed by feverishness and gradually worsening pain in the left side of the chest. The sputum was bloodstained, and there was bleeding from the nose. On the day of admission he vomited a little blood.

For as long as he could remember he had been subject to a local purpuric eruption whenever his skin was firmly stroked or pinched. During the years before admission his exercise tolerance had been restricted by breathlessness. He had been told that a chest radiograph taken three years after the dyspnoea began showed "healed miliary tuberculosis." and further radiographs during the following years were said to have shown no change. He was known to have a cardiac murmur: this had been described as a nonorganic pulmonary systolic murmur.

On admission his temperature was $99.4^{\circ} \mathrm{F}$. $\left(37.4^{\circ}\right.$ C.). There was widespread purpura, particularly on the trunk. Scratching the skin was promptly followed by a crop of purpuric spots along the scratch line. A few haemorrhagic herpetic vesicles were present on the upper lip and there were petechiae in the oral mucosa. The heart was enlarged (apex beat in sixth interspace just outside the mid-clavicular line). A harsh systolic murmur was audible over the whole precordium, and loudest in the fourth left interspace beside the sternum. Pulse regular (100 per minute). Blood pressure $130 / 70 \mathrm{~mm}$. of mercury. There were scattered rales in the lungs. The edge of the liver was palpable $2.5 \mathrm{~cm}$. below the costal margin, and the spleen could just be felt.

Investigations. - Chest radiograph: uniform, close stippling of lungs with "hard" miliary shadows. Electrocardiography: left ventricular preponderance. Blood : erythrocytes $3,750,000$ per c.mm.; haemoglobin $64 \%$; leucocytes 18,700 per c.mm. (neutrophils 15,300, eosinophils 100 , lymphocytes 1,400 , monocytes 1,800 , Türk cells 100 ); platelets 363,000 per c.mm. ; " target cells " present. Urine : protein + ; hyaline casts and occasional leucocytes and erythrocytes.
Progress.-Eight hours after admission the patient had severe melaena, followed by haematemesis. In spite of immediate blood transfusion he quickly died.

Necropsy.-The stomach contained about $600 \mathrm{ml}$. of freshly clotted blood, and there was a large amount of fresh blood in the lumen of the small intestine. The gastric and intestinal mucosa was severely autolysed and stained by blood and bile : no ulceration and no indisputable purpura or other source of haemorrhage was visible to the naked eye. The peritoneum was normal; there was no ascites. The liver $(1,800$ g.), pancreas (400 g.), and spleen (250 g.), a splenunculus (20 g.), and the kidneys (combined weight 450 g.) and adrenals (combined weight 45 g.) showed the typical appearances of severe diffuse amyloidosis. The iodine test gave no clear result, but on applying sulphuric acid some of the amyloid developed a muddy bluish-olive colour. The tongue was moderately enlarged; there were deep, clean folds between amyloid deposits in the mucosa of its posterior third, and its musculature was widely affected by amyloidosis. The mouth otherwise, and the salivary glands, pharynx, and oesophagus were normal. The nose, nasal sinuses, larynx, trachea and bronchi, and thyroid were normal. Small effusions were present in the pleural cavities, which were partly obliterated by old fibrous adhesions; adhesions bound the pleurae to the pericardium. The lungs were of normal size, but hyperaemic; they were studded throughout with firm, black nodules, uniformly about $0.35 \mathrm{~cm}$. in diameter, and most numerous in the hilar and central parts of each lobe. The pulmonary vessels were normal. The heart (500 g.) was hypertrophied, particularly the left ventricle; the myocardium looked otherwise healthy and no amyloid was evident; there was no septal defect. The valves, endocardium, and coronary vessels were normal; the pericardial sac contained a small excess of fluid but otherwise was normal. There was slight aortic atheroma. The urinary tract, prostate, seminal vesicles, and testicles were normal. The brain was normal. The skull, spine, thoracic cage, and pelvic bones, and the accessible skeletal muscles were normal. The tissues in general were dry ; there was no oedema. There was widespread purpura of the skin, with ecchymoses in some areas, particularly around the eyes and in the neck.

The lymph nodes throughout the body were grossly abnormal. The mesenteric nodes were enlarged, the biggest measuring 5 by 3 by $1.5 \mathrm{~cm}$; their cut surface was finely spongy, showing yellow or orange, opaque, soft material separated by fine, interlaced trabeculae. Similar appearances were seen in the juxta-aortic and iliac lymph nodes and in those of the mediastinum and neck; the superior vena cava was compressed by groups of greatly enlarged nodes. Extensive fresh haemorrhage was present in many of these mediastinal nodes, in some instances virtually destroying them.

Histology.-The distribution and appearance of the amyloid deposits in the most heavily involved organs, 


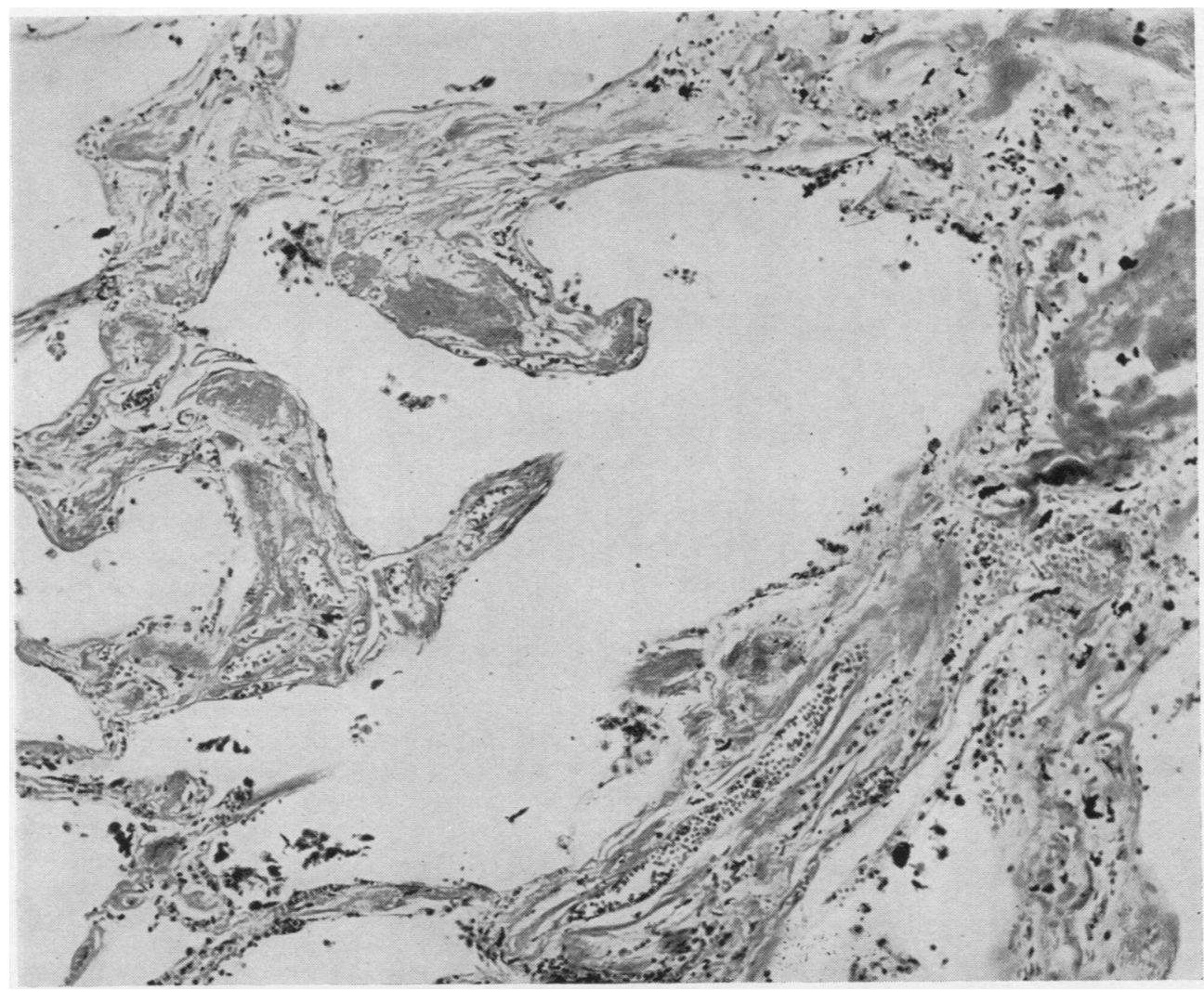
FIG. 7.-Case 5. Amyloidosis in alveolar septa of lung, at margin of a calcified amyloid nodule. Haematoxylin

which included the lymph nodes, were typical of that seen in classical cases of severe secondary amyloidosis and require no further description. The amyloid had strong affinity for Congo red, and for the most part gave a clearly metachromatic reaction with methyl violet. It gave a positive but not very intense periodicacid-Schiff reaction. In frozen sections abundant Sudanophil lipid was present in the amyloid in the spleen and to a less extent in the liver and a mesenteric lymph node, but not in other situations : only interstitial and pericapillary amyloid contained fat, that in the walls of larger blood vessels being free of demonstrable fat. In most tissues sectioned there was heavy amyloidosis of the walls of blood vessels, particularly small arteries and arterioles.

Sections of the stomach and small intestine showed widespread and severe amyloidosis in the mucosa and in submucosal blood vessels, with scanty pericellular amyloid in the muscle coats. In spite of severe autolysis it was clear that there was extensive recent haemorrhage in the mucosa, particularly in the immediate vicinity of arterioles showing heavy amyloid involvement. The source of bleeding in the haemorrhagic lymph nodes could not be determined, so severe was the destruction caused; there was well-advanced organization of the haematomas in some nodes.

The tongue was heavily affected, with amyloid deposits separating and destroying the muscle cells. No other skeletal musculature was found to be involved. Amyloid in the heart was confined to vessel walls ; the myocardium was hypertrophic : occasional small foci of advanced cytolysis were present. The nodules noted in the lungs macroscopically were areas of heavy amyloidosis of interalveolar septa (Fig. 7), with carbon pigmentation and focal calcification; in addition there was patchy amyloidosis of the larger blood vessels and in the walls of bronchi and some bronchioles, occasionally with small local haemorrhages.

The parenchyma of the brain and the vessels in it were free from amyloid, although some meningeal vessels and the choroid plexuses were heavily affected. The blood vessels of the skin showed severe amyloidosis, particularly the arterioles, and there were fresh haemorrhages around some vessels. Interstitial amyloidosis was present sparsely in the superficial zone of the dermis. Subcutaneous fat, and adipose tissue elsewhere in the body, was almost free from amyloidosis, 
although in some sections pericellular amyloid rings were found.

This case illustrates well that amyloidosis may occur without a recognizable predisposing cause and yet present the gross distribution of amyloid which is usually associated with secondary amyloidosis. The presence of macroglossia has commonly been stressed in the literature as a valuable diagnostic feature of primary amyloidosis : its presence should indeed draw attention to the possibility of amyloidosis, but one must remember that it is absent oftener than present in primary amyloidosis, and that it occurs, although rarely, in secondary amyloidosis.

The haemorrhagic manifestations are a puzzling feature of this case. The sudden onset and rapidly fatal outcome of the final gastro-intestinal haemorrhage suggested that the bleeding would prove to have come from a major, localized source: but a very thorough search failed to show any such source, and histological examination showed widespread gastrointestinal purpura in relation to severe amyloidosis of many small vessels. While a major lesion may indeed have been overlooked, the available evidence suggests that the fatal haemorrhage was purpuric in nature: if this is correct it remains to be explained how such sudden widespread haemorrhage could occur more or less synchronously from so many vessels affected by this essentially chronic alteration of their substance. Moreover, it is difficult to understand why the haemorrhage should be so much severer and more widespread in the alimentary tract than in other tissues with vascular amyloidosis of comparable degree, e.g., the bronchi. The extensive recent and fresh haemorrhages in some of the amyloid lymph nodes is a further remarkable and unexplained feature of this case. Finally, how is one to relate the terminal haemorrhagic state and its underlying vascular amyloidosis to the apparently very long history of purpura associated with trivial pressure on or stretching of the skin? The clinical history and the radiological and post-mortem findings together suggest that pulmonary amyloidosis had been present for at least 10 years, seemingly without detectable alteration in its extent : if this is the true interpretation of the observations, does it imply that amyloidosis had been present for considerably longer, and even for as long as the haemorrhagic tendency? Unfortunately, the onset of the latter cannot be dated from the available records. Most cases of generalized amyloidosis run their course within one to three years of the first symptom : oniy a few cases have been reported in which the course was considerably longer, and the longest survival on record is 14 years (Koletsky and Stecher, 1939).

Eisen (1946) and Mathews (1954) cited a small number of recorded cases of primary amyloidosis with severe gastrointestinal haemorrhage: such haemorrhage has usually been traced to erosive or frankly ulcerative lesions, which were not observed in the present cases (Cases 4 and 5) but might have been obscured by the severe autolysis in Case 5. Propp, Scharfman, Beebe, and Wright (1954) have discussed the association between primary amyloidosis and athrombocytopenic purpura, which they considered to be vascular in origin.

\section{Amyloidosis Associated with Disturbance of Endocrine Balance}

Case 6 : Amyloidosis Associated wiah Exophthalmis Ophthalmoplegia and Pretibial Myxoedema after Thyroidectomy for Hyperthyroidism.--A woman, aged 67, was admitted to hospital with myxoedema. Fourteen years earlier, after an attack of pneumonia, she began to lose weight. became breathless on exertion. nervous. and easily tired, and developed progressive bilateral staring exophthalmos. The menopause had been a year before the onset of these symptoms. Her family doctor gave her a course of thyroid extract, which greatly aggravated her condition. Eventually, four years after the onset of thyrotoxic symptoms, subtotal thyroidestomy was performed. Shortly after this she was told by her doctor that the surgeon had removed too much of the thyroid; he prescribed nothing. After the operation her right eye became normal, and it remained so : progressive protrusion of the left eye led within a few months to severe exophthalmos, with paralysis of the external ocular muscles. At about the same time she began to have severe pain in the hips, lumbar region, knees, and wrists; this continued to trouble her considerably throughout the rest of her life. Her energy and strength failed: the hair of her scalp and eyebrows became thin. Two months before admission to hospital she developed diarrhoea, and this persisted, with several fluid motions daily. Dried thyroid extract was given soon after the diarrhoea began: she had not had thyroid since the thyroidectomy ; only half a grain $(0.03 \mathrm{~g}$.) daily was prescribed, and this had no apparent effect. She was therefore referred to hospital. where she was admitted immediately.

On admission she presented the typical picture of severe myxoedema, together with left-sided exophthalmic ophthalmoplegia and severe pretibial myxoedema. In addition there was a palpable tumour in the left hypochondrium, and severe osteoarthritis of the hips and knees. There was no evidence of heart failure. Blood pressure $140,90 \mathrm{~mm}$. of mercury. The liver and spleen were not palpable.

Investigations.-Urine : protein + . Blood: erythrocytes 3,110,000 per c.mm.: haemoglobin 9.4 g. per 100 
ml.; leucocytes 7,100 per c.mm. (myelocytes 35 , metamyelocytes 180 , neutrophils 4,685 , eosinophils 35 , lymphocytes 1,280 , monocytes 815 , plasma cells 35 , Türk cells 35$)$. Erythrocyte sedimentation rate $\mathbf{7 0}$ $\mathrm{mm}$. in one hour (Westergren). Blood urea $43 \mathrm{mg}$. per $100 \mathrm{ml}$. Basal metabolic rate minus $35 \%$.

Progress.-Thyroid extract was given by mouth, half a grain $(0.03$ g.) three times a day. Five days after admission the patient collapsed suddenly; she remained semiconscious from then until her death three days later. During this time there was gross and uncontrollable disturbance of serum electrolytes.

The serum potassium concentration ranged between $21.4 \mathrm{mg}$. and $13 \mathrm{mg}$. per $100 \mathrm{ml}$., the lowest figure being found on the day of death, in spite of parenteral and oral administration of potassium salts.

Necropsy.-The pretibial tissues had the classical appearances of myxoedema. Similar myxoedematous material filled the left orbit, pushing the bulb forward. The thyroid was represented by three rounded nodules of fibrotic tissue, each about $0.5 \mathrm{~cm}$. in diameter. The heart (530 g.) was enlarged by hypertrophy and dilatation of the ventricles. The hypertrophic myocardium looked healthy and the valves, endocardium, and pericardium were normal. The coronary vessels were normal. There was atheromatous ulceration of the abdominal aorta. The main bronchi of the lower lobe of each lung showed fusiform dilatation, with some chronic suppuration and peribronchial fibrosis. The rest of the lungs, the pleurae, and the upper respiratory tract were normal. The mouth, salivary glands, and tonsils were normal ; the tongue was not enlarged. The oesophagus, stomach, intestines, liver (1,450 g.), biliary tract, pancreas, and spleen (110 g.) appeared healthy. There was no lymphadenopathy. The adrenals (combined weight 10.5 g.) were small and pale.

Both kidneys (right, 165 g.; left, 385 g.) showed narrowing of the cortex and fine granularity of their outer surface. A typical, infiltrating Grawitz tumour ( 8 by 7 by $6 \mathrm{~cm}$.) occupied the middle third of the left kidney; no metastases were found anywhere in the body. The urinary tract and reproductive organs were normal. The brain and the accessible parts of the skeleton were normal ; the hip joints and knees were not opened, but there was external evidence of osteoarthritis.

Histology.-Patchy or severe amyloidosis affected small arteries, arterioles, and venules, and occasionally capillaries, in all the tissues sectioned, except the brain, which was histologically normal. The spinal cord was not examined. The degree of vascular involvement differed from organ to organ : it was particularly widespread and severe in the myocardium and gastrointestinal tract and around the adrenals, and was scanty in the liver, kidneys, and spleen. The parenchyma of the solid organs was not affected. Pericellular amyloid was most abundant in the adipose tissues, but was present also in many small areas of myocardium and in the muscularis mucosae of the stomach and intestines and, to a less extent, in their main muscle coats. Amyloid deposits were present in the interstices of the connective tissue of the gastrointestinal mucosa and of the tongue between its surface epithelium and its musculature. Skeletal muscle, including that of the tongue, was scarcely involved, and virtually no amyloid was found in skin or bone marrow. The haemopoietic marrow showed erythroid and myeloid hyperplasia; there was no evidence of leukaemia. The pretibial myxoedema was histologically typical : similar myxoedematous infiltrate was present in the left orbital tissues. There was no cellular infiltration of any sort in the orbital tissues, including the ocular muscles. No myxoedematous infiltrate was found elsewhere. Minimal mucoid degeneration of myocardial fibres was seen.

The thyroid remnants were fibrotic; the surviving acini were lined by tall epithelium, often with much papillary ingrowth, and only a little, poorly stained colloid was present. The pituitary showed a frank reduction in the proportion of acidophil and basophil cells. Many chromophobe cells were uncommonly large; their cytoplasm sometimes contained one or two large vacuoles; in places they formed rosette-like groups, and in these they assumed a columnar or wedge shape. Calcified, concentrically laminated spherules were scattered through the anterior lobe. There were areas of hyaline thickening of the connective-tissue framework of the gland; amyloid was found only in arteriole walls, and was sparse there. The adrenal cortex was narrow and the zona glomerulosa was ill-defined or lacking; amyloid was confined to the walls of arterioles and of the central veins. The histological findings in the pituitary and adrenals corresponded very closely to those described by Brewer (1951) in his case of uncomplicated myxoedema.

Histochemical Findings.-The pretibial and orbital myxoedema infiltrate gave a positive reaction with periodic-acid-Schiff, stained metachromatically with toluidine blue and was mucicarminophil. It was strongly acid, taking up methylene blue at $p \mathrm{H} 3$. In sections which had been incubated with hyaluronidase there was no appreciable difference in the reaction with periodic-acid-Schiff but metachromasia with toluidine blue was considerably less apparent; incubation with diastase had no effect. These results correspond to those found by Brewer (1951) on investigating the mucoid infiltration in the tongue in his case of myxoedema, and they were obtained by the methods which he described: they suggest that the myxoedema infiltrate may be a mixture of hyaluronic acid and a strongly acid polysaccharide, possibly of the nature of chondroitin sulphuric acid.

The amyloid deposits in this case had considerable affinity for Congo red and stained metachromatically with rosanilin dyes. It was noticed that in parts of the heart small clumps of interstitial amyloid gave a strong positive reaction with periodic-acid-Schiff, appeared to stain metachromatically with toluidine blue and were mucicarminophil, while most of the 
amyloid elsewhere gave only a weak reaction with periodic-acid-Schiff and did not stain with mucicarmine or metachromatically with toluidine blue. In the strongly reacting areas there was abundant, but not pathologically excessive, connective-tissue ground substance, which stained metachromatically with toluidine blue and was mucicarminophil and unusually acid, taking up methylene blue at $p \mathrm{H} 3$. Incubation with hyaluronidase greatly diminished the toluidineblue metachromasia, the mucicarminophilia and the reaction with periodic-acid-Schiff, in both the ground substance and the amyloid. Neither hyaluronidase nor diastase had any effect on the staining of the amyloid with rosanilin dyes or with Congo red. These observations suggest that the anomalous staining of the amyloid was due to its being bathed in the apparently somewhat abnormal connective-tissue mucoid: they do not, of course, prove any derivation of the one substance from the other.

Of the four conditions which were associated with amyloidosis in this case-osteo-arthritis, renal carcinoma, bronchiectasis, and the post-thyroidectomy state-it seems reasonable to exclude the first two from consideration as possible predisposing causes. Amyloidosis has not been observed to occur with unusual frequency in cases of osteoarthritis, in contrast to the known predisposition to amyloidosis in cases of rheumatoid arthritis (Reece and Reynolds, 1954). The presence of the renal carcinoma may be considered incidental. Amyloidosis may occur as a complication of cancers, but ordinarily this association is only seen with slowly progressive growths with ulceration and chronic infection (Dahlin, 1949); there is no known predisposition to amyloidosis in cases of other neoplasms, with the exception of myelomatosis, which is in a special category in this respect as amyloidosis occurs in $10 \%$ or more cases (Snapper, Turner, and Moscovitz, 1953). Although plasma cells were found in the peripheral blood in this case, and there was a sparse accumulation of plasma cells in the haemopoietic marrow and in the bronchiectatic lesions, nothing suggesting myeloma was found anywhere.

Bronchiectasis is one of the recognized predisposing causes of amyloidosis. The resulting amyloidosis usually has the distribution of classical secondary amyloidosis ; occasionally, however, the distribution is that which is more characteristically seen in amyloidosis without a recognized cause. Bronchiectasis certainly cannot be excluded as a cause, and possibly the sole predisposing cause, of amyloidosis in this case.

In addition, this patient was at an age when primary senile amyloidosis begins to appear. Hüsselmann (1955) found that about $15 \%$ of men and $10 \%$ of women over 70 have amyloidosis : this senile amyloidosis is similar in its manifestations to primary amyloidosis of younger people, particularly in its predilection for the cardiovascular tissues, and it has a special tendency to affect the heart predominantly.

The relation between the post-thyroidectomy state in this case, with its disturbance of pituitarythyroid function, and the amyloidosis is debatable. It is possible that hypothyroidism enhanced amyloid formation. There seems to be little doubt from the patient's history that she had previously had hyperthyroidism. Attempts to get information about her condition from the hospital where the thyroidectomy was performed were unsuccessful, and requests for access to sections of the surgical specimen were refused, so that it has been impossible to discover whether there was histological evidence of amyloidosis at that time. This is particularly unfortunate in view of the possibility that there might have been evidence of amyloidosis antedating the hypothyroidism. The association of amyloidosis and hypothyroidism was observed also in the case described next in this paper, but appears to have been described only once before in the literature (Case Records of the Massachusetts General Hospital, 1956). As in the present case, the Massachusetts General Hospital patient was a woman who had what was in effect a total thyroidectomy for hyperthyroidism. Six years later, when aged 30 , she was found to have amyloidosis : necropsy showed involvement of lymph nodes, salivary glands, skeletal muscle, intestines, and lungs, and no cause for amyloidosis was found. Exophthalmos was never present and myxoedematous deposits were not noted in the Massachusetts case; the patient had received some maintenance treatment with thyroid.

There is experimental evidence that hormones can influence amyloid formation. Peräsalo and Latvalahti (1954) found that corticotrophin and, to a less extent, cortisone enhanced amyloid production in mice receiving caseinate injections; castration had a similar effect, while testosterone was without obvious influence; thyroidectomy also encouraged the development of amyloidosis, while thyroid extract appeared to have some inhibitory effect upon it. Uotila, Peräsalo, and Vapaavuori (1955) found that growth hormone had no effect on the development of experimental amyloidosis, whereas thyrotrophin accelerated it. Confirmation of these findings would be a considerable advance in knowledge of the factors concerned in amyloid formation, particularly in relation to cases such as this one and the next. Meantime, it is interesting to speculate about the 
possible role of thyrotrophin, diverted from its proper sphere of activity by the absence of thyroid tissue, in the development of exophthalmos, pretibial myxoedema, and amyloidosis in this case.

Amyloid is an atypical and predominantly protein substance probably containing a small proportion of polysaccharide, which is usually said to be chondroitin sulphuric acid. Chondroitin sulphuric acids, hyaluronic acids, and protein are constituents of the mucoid material of myxoedema infiltrates (Brewer, 1951; Bruns, 1955). The possibility of a relationship between amyloid and myxoedema mucoid might repay study. It is interesting to observe the similar distribution of the amyloid deposits in some cases and of myxoedema infiltrates. This is reflected, for instance, in the diagnostically important observation that the two commonest causes of macroglossia are amyloidosis and myxoedema. Brewer (1951) showed that the "basophilic degeneration " of the myocardial cells, which is particularly frequent and extensive in myxoedema, is due to deposition of a mucoprotein distinct from that of the interstitial myxoedema infiltrates. The presence of this myocardial mucoid degeneration and of the chemically distinct myxoedema infiltrate in other tissues indicates the occurrence in hypothroidism of two pathological alterations in the utilization of polysaccharides : a third alteration, resulting in the formation of amyloid, might be mediated similarly, or amyloid might even result by transformation of myxoedema infiltrate.

As only the Massachusetts General Hospital case and the two cases in this paper have been recorded, so far, it must be very rare for clinical evidence of endocrine dysfunction to be a feature of the antecedent history of cases of amyloidosis. It is clear that neither hypothyroidism nor any other endocrine disorder can be held responsible, on the evidence available, for any considerable proportion of cases of amyloidosis in which no obvious predisposing cause is found. The most that can be said is that these three cases illustrate a state of affairs which resembles that observed experimentally by the Finnish workers, and that they are an encouragement to further investigation along these lines. Further research, however, should be directed as much to investigating changes in polysaccharide and protein metabolism and the role of hyperglobulinaemia, plasma-cell proliferation and immunological disturbances as to the study of hormonal influences. The problem of amyloidosis is clearly a complex one, in which many factors are concerned.
Haemmerli (1954), on the basis of clinical and post-mortem observations, suggested that hypothyroidism due to amyloid deposition in the thyroid might contribute to the picture of primary amyloidosis, and he advised considering the use of thyroid extract in treatment. While there is in fact only rarely any considerable degree of thyroid amyloidosis in these cases, Haemmerli's suggestion would seem to be worth following up, in view of the experimental findings already referred to and of the clinical observations in the next case. Care would obviously be necessary in giving thyroid extract to patients with serious cardiac disability, and the need for thorough investigation before and during treatment is self-evident.

To summarize the case described above, it can be said that the development of amyloidosis may have been determined primarily by chronic suppurative bronchiectasis, by the patient's age (senile amyloidosis), or by the pituitary-thyroid disturbance, or by the combination of any of these. The distribution of the amyloid deposits, with predominant involvement of tissues which are characteristically involved in cases of amyloidosis without recognized cause (" primary amyloidosis"), is of no differential significance, for the same distribution is found in a proportion of cases of amyloidosis occurring as a complication of the classical predisposing causes (see Cases 11 and 12, below).

Case 7 : Amyloidosis Associated with Myxoedema after Treatment of Hyperthyroidism with Radioactive Iodine.-A man, aged 27, developed severe hyperthyroidism, with a large goitre and exophthalmos. He refused surgical treatment and failed to co-operate in treatment with thiouracil. He was advised to have treatment with radioactive iodine and this was carried out. Inadvertently, he received three times the calculated dose. No lymphadenopathy, hepatosplenomegaly, or macroglossia was present at that time. He was next seen two years later, when he presented the picture of severe hypothyroidism. His basal metabolic rate was minus $32 \%$. In addition, the liver and spleen were hard, and palpable $2 \mathrm{~cm}$. below the costal margin ; he had generalized enlargement of superficial lymph nodes, and macroglossia of such degree that he could not close his jaws.

Investigations.-Slight proteinuria. Serum albumin 2.8 g. per $100 \mathrm{ml}$.; globulin 2.2 g. Erythrocytes $4,100,000$ per c.mm.; haemoglobin 10.2 g. per $100 \mathrm{ml}$. Chest radiograph : slight increase of transverse dimension of heart; otherwise normal. Electrocardiography: low voltage in all leads.

Biopsy of an axillary lymph node (Fig. 8) and of the tongue showed widespread amyloid deposits. The amyloid stained metachromatically with methyl violet and had considerable affinity for Congo red. 


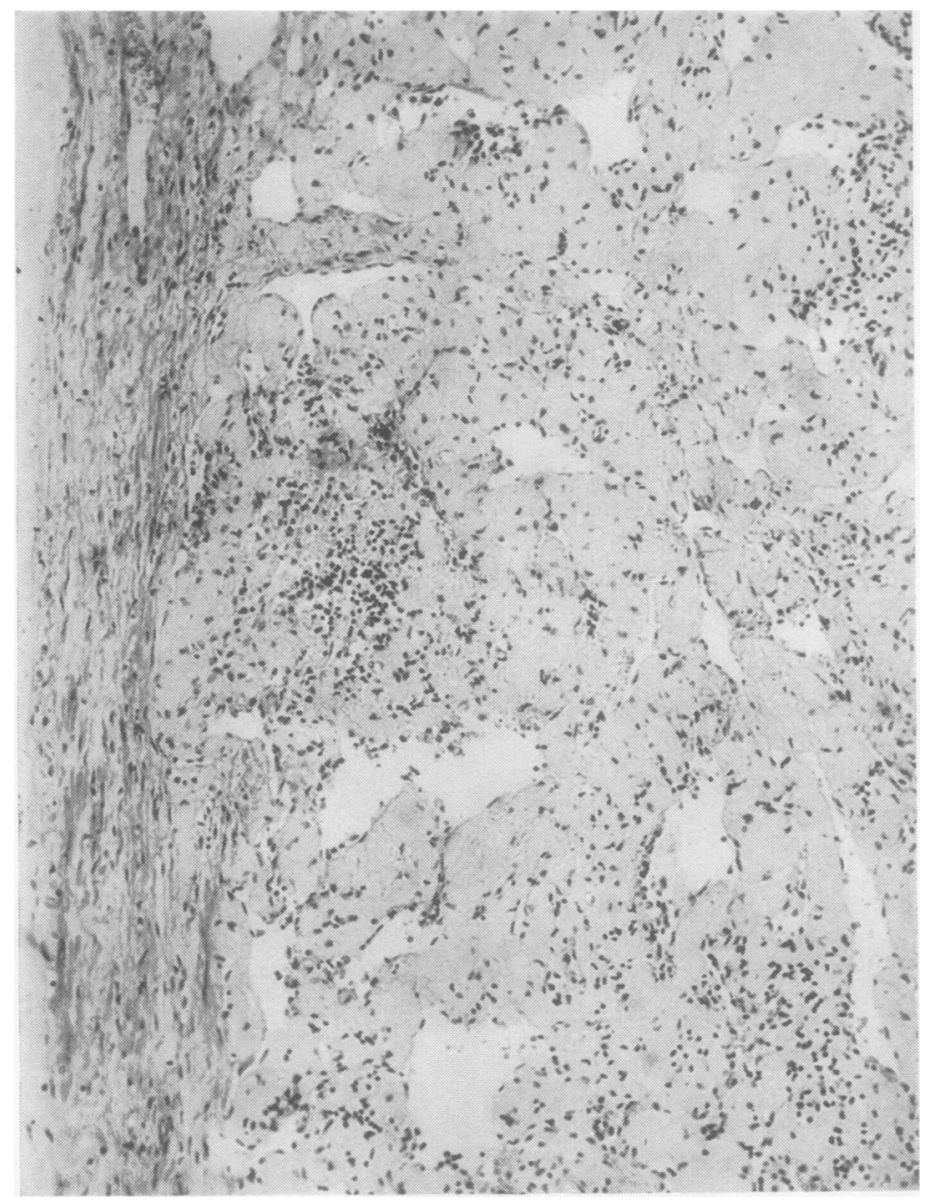

FIG. 8.-Case 7. Amyloidcsis of a lymph node. Haematoxylin and eosin.

passu is itself suggestive of a relation between the two, and a relationship is supported by the regression of the amyloidosis when the hypothyroidism was brought under control by substitution therapy. It is obviously impossible to determine how far the manifestations observed during the phase of uncorrected myxoedema were due to hypothyroidism per se and how far to amyloidosis; equally, it is impossible to determine how far the disappearance of these manifestations was the result simply of recovery from the usual effects of hypothyroidism and how far it represented regression of amyloidosis.

\section{Localized Amyloidosis}

Case 8: Amyloidosis of Unknown Cause Apparently Confined to the Mammary Areolae and the Vulva.A medical woman, aged 24 , who had never had any serious or chronic illness, volunteered to take part in an investigation in normal people of the haemostatic activity of Congo red and of the uptake and excretion of the dye. She was given an intravenous injection of $10 \mathrm{ml}$. of $1 \%$ solution of Congo red: the usual calculation showed $65^{\circ}$ o of the dye still to be present in the blood stream $60 \mathrm{mi}$ nutes after the injection. Next morning she noticed a red circle around the areola of each breast. Examination showed a vivid red zone.

Progress.-The patient was treated with thyroid extract by mouth, and a maintenance dose of 4 grains (0.26 g.) daily kept his basal metabolic rate within normal limits. During the following six months the general mental and physical stigmata of myxoedema regressed satisfactorily. The tongue decreased in size until it was not obviously larger than normal. The liver and spleen ceased to be palpable. and the superficial lymph nodes became smaller although they remained palpable and firm. The size of the heart on radiography and the electrocardiograph returned to normal. When last seen, 12 months after starting treatment with thyroid extract, he felt very well. The urine still contained a trace of protein; the serum albumin concentration was $3.8 \mathrm{~g}$. per $100 \mathrm{ml}$. and globulin $2.3 \mathrm{~g}$. The lymph nodes continued to decrease in size.

No cause for amyloidosis was found in this case, unless it was a result of the gross destruction of the thyroid by radio-active iodine. The development of amyloidosis and myxoedema pari about $1.5 \mathrm{~cm}$. wide, in the skin of each breast, sharply defined at its periphery and merging centrally with the areola. The areolae were black and completely smooth ; their skin appeared healthy, but was fixed almost immovably to an underlying disc of tough, remarkably resilient tissue. This disc had a thin but distinct edge which coincided precisely with the outer edge of the reddened zone : it was possible to press one's fingers deep under the disc, which seemed to be uniformly about $5 \mathrm{~mm}$. in thickness, except at its tapered edge ; on compressing it from side to side it bent up like the rubber sole of a slipper, springing back at once to its previous shape when released. The nipples were normal. The skin of the axillae, vulva, and perineum was melanotic, although considerably less so than the areolae. The labia minora were well developed. but not abnormally large or thick ; each contained several irregular, indurated plaques, up to $1 \mathrm{~cm}$. across, which occupied the full thickness of the labium and were adherent to its skin: on the amelanotic, medial side of the labium the skin immediately over the plaques showed the same peculiar red 
colour as was seen on the breasts, and the hymen was similarly coloured. Clinical examination showed no other abnormality; no unusual pigmentations or other lesions were found elsewhere in the skin.

The patient said that the indurated plaques had been present ever since her breasts developed at puberty, about 11 years before, and that the areolar skin had always been smooth, relatively firmly attached to the plaques, and uncontractile, whereas the nipples were erectile. She herself attributed the intense melanosis of the areolae, axillae, and vulva to a former habit of occasionally taking large doses of hexoestrol by mouth in order to delay menstruation. At the time of the Congo red injection she had not tried to interfere with menstrual function for almost two years, and her cycle was perfectly normal.

Investigations.-Urine, blood picture, erythrocyte sedimentation rate, plasma proteins, serum electrolytes, and chest radiographs were normal. A biopsy of the red zone on one breast showed that the underlying plaque consisted of amyloid, intensely stained in situ by Congo red. Microscopically, the amyloid formed a dense, homogeneous mass, sharply demarcated from the normal underlying adipose tissue ; only a shallow zone of the superficial part of the dermis was unaffected. No muscle was seen in the sections, other than that of the blood vessels, which were normal; epidermal appendages were also absent, although the epidermis itself appeared to be normal apart from intense pigmentation with melanin at the edge of the areola. The amyloid stained metachromatically with methyl violet ; there was no cellular reaction of any sort around it.

Progress.-The Congo red staining of the areolae faded gradually, but traces were still visible for more than five months. The patient has kept perfectly well during the five years which have passed since the amyloid nature of the plaques was discovered. She has been married for three years, and has two children, both of whom were breast-fed without difficulty. In view of the introital induration hymenectomy was performed before marriage ; the presence of amyloid in the hymen was confirmed microscopically, and very faint traces of the original Congo red staining were recognizable although almost two years had passed since the injection. The patient has noticed no difference in the amyloid plaques in the breasts, although those in the labia are no longer palpable.

This case appears to be unique, not only in the way in which it came to light but in the distribution of the deposits also. The manifestations do not correspond to any recognized form of cutaneous amyloidosis (Andrews, 1954), whether localized or as a manifestation of generalized amyloidosis. There is clearly no evidence of any generalized disease in this patient. It is noteworthy that the subareolar discs were known by her to be present before she took oestrogens : a causal relation betweeen this self-medication and the amyloidosis can be set aside. Other varieties of localized amyloidosis have been mentioned briefly elsewhere (Symmers, 1956).

\section{Amyloidosis Associated with Rheumatic Heart Disease}

Case 9: Amyloidosis and Recurrent Rheumatic Pancarditis.-A woman, aged 43, was admitted to hospital with acute polyarthritis. She had had rheumatic fever at the ages of $16,20,32$, and 36 , and severe mitral and aortic valvular lesions were present. Acute heart failure developed, and she died five days after admission.

Necropsy showed acute rheumatic pericarditis and endocarditis, with old rheumatic disease of all four valveś, and generalized chronic venous congestion. Histological examination showed acute rheumatic lesions in all parts of the heart. There was amyloid macroglossia, widespread amyloidosis of blood vessels throughout the body, and pericellular amyloidosis in the musculature of the alimentary tract and to a slight degree in the myocardium. The amyloid deposits in the myocardium appeared to be independent of the Aschoff bodies, and of the old interstitial scars. Except for involvement of their larger blood vessels the kidneys, liver, spleen, and adrenals were free from amyloid.

Case 10 : Amyloidosis and Recurrent Rheumatic Carditis and Polyarthritis.--A woman, aged 53, was admitted to hospital because of fever, polyarthritis, and anaemia. Her only previous illness was rheumatic fever at the age of 20 , and this had left her with aortic-valve incompetence. A year before admission, and just after the menopause, she began to have recurrent attacks of painful swelling of the knees. ankles, wrists, and hands, and her health steadily failed. Aspirin regularly relieved the pain. On admission, examination showed painful swelling, redness, and effusion in the knee, wrist, and finger joints ; there was no deformity. Gross aortic-valve incompetence was present ; there was no evidence of heart failure. Temperature $100^{\circ}$ F. $\left(37.8^{\circ}\right.$ C. $)$. Urine: protein ++ Blood: erythrocytes 2,500,000 per c.mm., haemoglobin 7.7 g. per $100 \mathrm{ml}$., leucocytes $13,100(80 \%$ neutrophil polymorphs). Blood cultures sterile. Wassermann and gonococcal complementfixation tests negative. Serum antistreptolysin titre 20 units per ml. Four days after admission she died suddenly.

Necropsy showed gross scarring with calcification of the aortic valve, which was stenotic and incompetent ; the mitral valve showed less severe old rheumatic disease. The left ventricle was much hypertrophied and dilated. There were old pericardial adhesions and severe coronary atheroma. Widespread amyloidosis was present, affecting predominantly the parenchyma of the kidneys, liver (Fig. 9), and spleen. Occasional Aschoff bodies (Fig. 10) and extensive old interstitial fibrosis were found in the myocardium.

These two cases illustrate the association of rheumatic fever and amyloidosis; in one case the 


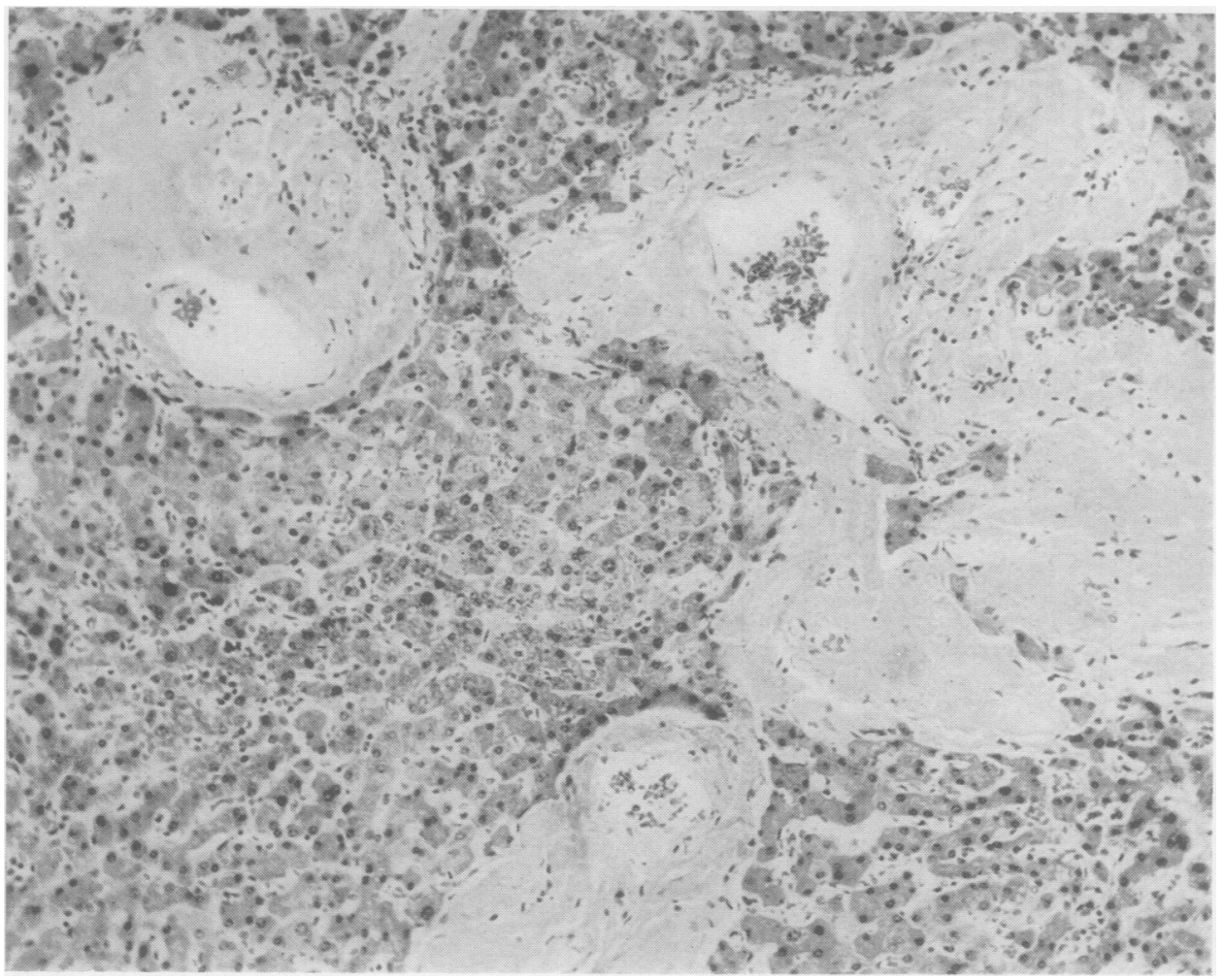

FIg. 9

FIG. 9.-Case 10. Amyloidosis of hepatic portal tracts. Haematoxylin and eosin. $\times 130$.

FIG. 10.-Case 10. Aschoff body. Haematoxylin and eosin. 180.

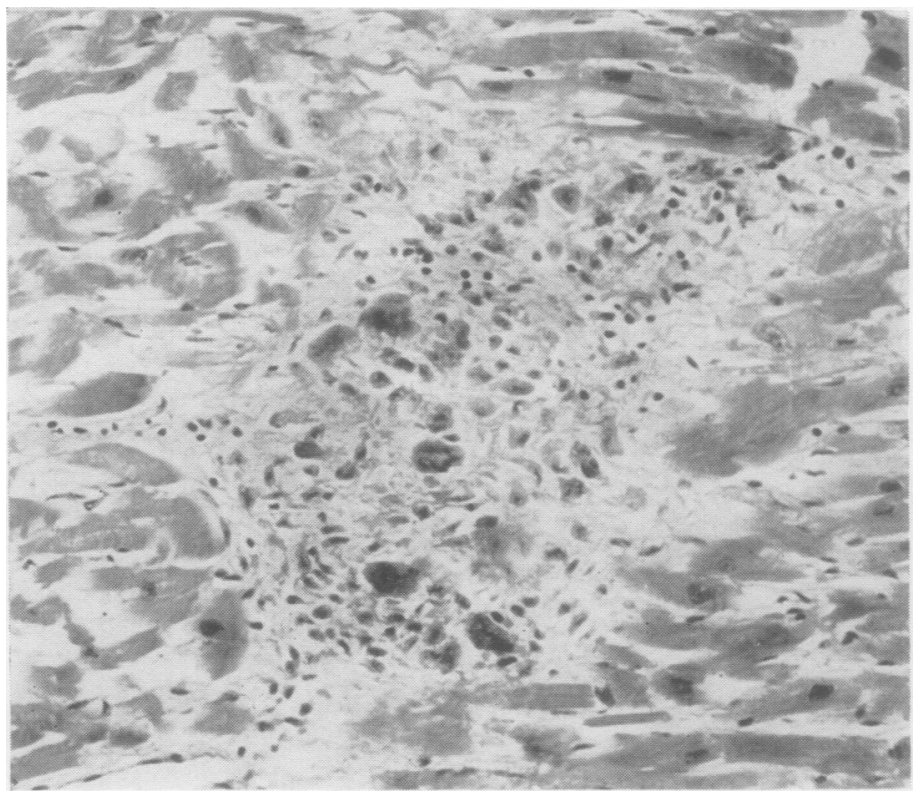

FIG. 10 
distribution of the amyloid deposits was that commonly seen in primary amyloidosis and in the other the picture was that of classical secondary amyloidosis. Amyloidosis is seldom found in association with rheumatic fever or its sequelae, but such cases have been described from time to time, for instance, by Wilks (1856), Beattie (1906), Dillon and Evans (1942), and Jackson (1954). Amyloidosis is much oftener seen as a complication of rheumatoid arthritis (Reece and Reynolds, 1954). The arthritis in the second of these two cases was considered on clinical grounds to be rheumatic in type rather than rheumatoid.

The association of amyloidosis with rheumatic fever has been said to suggest a relationship between amyloidosis and the so-called collagen diseases (Jackson, 1954); this scarcely seems to warrant describing any form of amyloidosis as a "collagen disease," and the relationship may prove to be tenuous or even illusory.

\section{Two Unusual Cases of Amyloidosis Associated with} Chronic Infection

Case 11 : Predominantly Cardiovascular and Lingual Amyloidosis Associated with Chronic Suppurative Osteomyelitis. - A man, aged 36, was admitted to hospital in the last stages of congestive heart failure ; he died two days later. He had had chronic suppurative osteomyelitis of the left femur for 14 years, with constant purulent discharge from two sinuses : the condition had caused him little inconvenience and he had declined to have any treatment, except occasionally to allow removal of small protruding sequestra. A year before his death he began to develop symptoms of congestive heart failure and his tongue was noted to be enlarging. No cause for the cardiac condition could be found clinically, and it proved to be refractory to all forms of treatment. A series of electrocardiographs showed a progressive fall in voltage in all leads during the last 10 months of his life. The Wassermann and Kahn tests were negative.

Necropsy showed severe chronic suppurative osteomyelitis of the femur, with large sequestra and several ramifying sinuses. There was no evidence of tuberculosis. The heart was enlarged and showed widespread amyloidosis. Lingual amyloidosis and amyloid plaques in the intestines were present. Histologically, there was extensive amyloidosis of blood vessels and of the musculature of the heart, tongue, alimentary tract, and abdominal wall. There was minimal amyloidosis of the liver, spleen, kidneys, and lungs. The amyloid stained metachromatically with methyl violet and showed a moderate affinity for Congo red.

Case 12 : Predominantly Cardiovascular Amyloidosis Associated with Chronic Actinomycosis.-A woman, aged 27, had appendicectomy in 1939 for acute suppurative appendicitis. The laparotomy wound did not heal fully, and a faecal fistula devel- oped. Five months later the fistula was excised and right-sided colectomy was performed. This was followed by the development of multiple abscesses, with sinuses and fistulae in the abdominal wall. A year after the appendicectomy an abscess formed in the right lung and was followed by pleural empyema and bronchopleural fistula. Actinomyces (israeli) was repeatedly isolated from pus from the abdominal and thoracic lesions. The patient became grossly emaciated and cachectic, and 19 months after the appendicectomy she died of actinomycotic meningitis. There was no clinical evidence of amyloidosis.

Necropsy showed extensive abdominal and thoracic actinomycosis, with terminal pyaemia and meningitis. There was no macroscopical evidence of amyloidosis, but sections showed very extensive pericellular amyloidosis of the myocardium, in the musculature of the stomach and intestines, in the myometrium and in the abdominal muscles. There was heavy involvement of the walls of arteries, arterioles, and veins in all the tissues sectioned, but no amyloid deposits were found in the parenchyma of the liver, kidneys, or spleen. The amyloid had little affinity for Congo red, and it showed metachromasia with methyl violet only in some areas.

These two cases illustrate that amyloidosis occurring with such a well-known predisposing condition as chronic suppuration can present the distribution which is usually associated with primary amyloidosis : the converse was illustrated by Case 5. While such observations do not prove the identity of all forms of amyloidosis, they indicate that the distribution of the deposits is not a sufficient criterion by which to attempt to distinguish different varieties.

\section{Summary}

Twelve cases of amyloidosis are described.

Five are cases of generalized amyloidosis without apparent predisposing cause (" primary amyloidosis") : heart failure, peripheral neuropathy, goitre, haemorrhage, and pulmonary lesions were among the presenting manifestations.

Two cases of amyloidosis associated with severe hypothyroidism following treatment for hyperthyroidism are reported, and the possible significance of the hormonal disturbance is discussed.

Amyloidosis, confined to the mammary areolae and vulva, was found unexpectedly in an otherwise healthy young woman.

Two cases of amyloidosis associated with recurrent rheumatic carditis are described. In one the distribution of the amyloid was that usually seen in primary amyloidosis; in the other it was that seen in classical cases of secondary amyloidosis.

Two cases of amyloidosis in patients with 
chronic septic lesions (osteomyelitis; widespread actinomycosis) are reported in which the amyloid was predominantly deposited in the cardiovascular system, almost completely sparing the organs usually affected in secondary amyloidosis.

Amyloidosis occurring without an apparent predisposing cause, that is, primary amyloidosis, including the predominantly cardiovascular senile amyloidosis, is probably commoner than secondary amyloidosis, particularly now that the incidence of the latter has been so much reduced by advances in the treatment of the diseases which formerly were its most frequent predisposing causes. Many more cases will have to be recognized during life if the nature of amyloidosis and the possibilities of developing methods of treating it are to be adequately investigated.

I am indebted to many colleagues for permisison to include accounts of their cases in this paper. In particular, I should like to thank Dr. A. P. Barter, Dr. P. B. S. Fowler, Dr. D. R. Humphreys, Dr. A. C. F. Ogilvie, Dr. N. S. Plummer. Dr. W. H. Russell Rees, Dr. J. H. Shore, and Dr. C. B. I. Willey for their help.

My thanks are also due to Mr. Allan Ayres and Mr. Frank Humberstone for technical assistance. and to $\mathrm{Mr}$. K. W. Iles for help in preparing the photomicrographs.
REFERENCES

Andrews, G. C. (1954). Diseases of the Skin for Practitioners and Students, 4th ed., pp. 579-583. Saunders, Philadelphia and Students,

Beattie, J. M. (1906). Brit. med. J., 2, 1444

Brewer, D. B. (1951). J. Path. Bact., 63, 503

Bruns, G. (1955). Zbl. allg. Path. path. Anat., 93, 246

Case Records of the Massachusetts General Hospital (1956). New Engl. J. Med., 254, 226.

Dahlin, D. C. (1949). Ann. intern. Med., 31, 105.

Dillon, J. A., and Evans, L. R. (1942). Ibid., 17, 722

Drapiewski, J. F., Sternlieb, S. B., and Jones, R. (1955). Ibid., 43, 406

Eisen, H. N. (1946). Amer. J. Med., 1, 144.

Gunnar, R. M., Dillon, R. F., Wallyn, R. J., and Elisberg, E. I. (1955). Circulation (N.Y.) 12, 827.

Haemmerli, A. (1954). Schweiz, med. Wschr., 84, 1262

Hüsselmann, H. (1955). Virchows Arch. path. Anat., 327, 607.

Jackson, A. (1954). Arch. intern. Med., 93, 494.

King. F. H., and Oppenheimer, G. D. (1948). Ann. intern. Med., 29 374.

Koletsky, S., and Stecher, R. M. (1939). Arch. Path. (Chicago), 27, 267

Lubarsch, O. (1929). Virchows Arch. path. Anat., $271,867$.

Mathews, W. H. (1954). Amer. J. med. Sci., 228, 317

Muehrcke, R. C. Pirani, C. L. Pollack, V. E., and Kark, R. M. (1955). Guy's Hosp. Rep., 104, 295.

Peräsalo, O., and Latvalahti, J. (1954). Acta path. microbiol. scand. 34, 208 .

Propp, S., Scharfman, W. B., Beebe, R. T., and Wright, A. W. (1954). Blood 9, 397

Reece, J. M., and Reynolds, T. B. (1954). Amer. J. med. Sci., 228. 554

Ritama, V., and Björkesten, G. af (1954). Ann. Med. intern. Fenn., 43. 152.

Snapper, I., Turner, L. B., and Moscovitz, H. L. (1953). Multiple Myeloma, pp. 85-94. Grune and Stratton, New York.

Soyka, J. (1876). Prag. med. Wschr., 1, 165

Symmers, W St C (1956). Journal of Clinical Pathology 9, 187.

Uotila, U., Peräsalo, $O$, and Vapaavuori, M. (1955), Acta path. microbiol. scand., 37, 322 .

Walker. G. A. (1942). Surg. Gynec. Obstet., 75, 374

Wild, C. (1886). Beitr. path. Anat., 1, 175.

Wiley, A. T., Teeter, R. R., and Schnabel, T. G. (1951). Med. Clin. Amer. 35, 1841 .

Wilks, S. (1856). Guy's Hosp. Rep., 17, (Series 3, 2) 103. 